\title{
Body mass index in a US national sample of Asian Americans: effects of nativity, years since immigration and socioeconomic status immigration and socio- economic status
}

DS Lauderdale and PJ Rathouz

Correction to: International Journal of Obesity (2000) 24, $1188-1194$.

International Journal of Obesity (2002) 26, 1522. doi:10.1038/sj.ijo.0802147

Following publication of the above paper, the author has identified errors in Figures 1 and 2. The correct versions of these Figures are reproduced below.

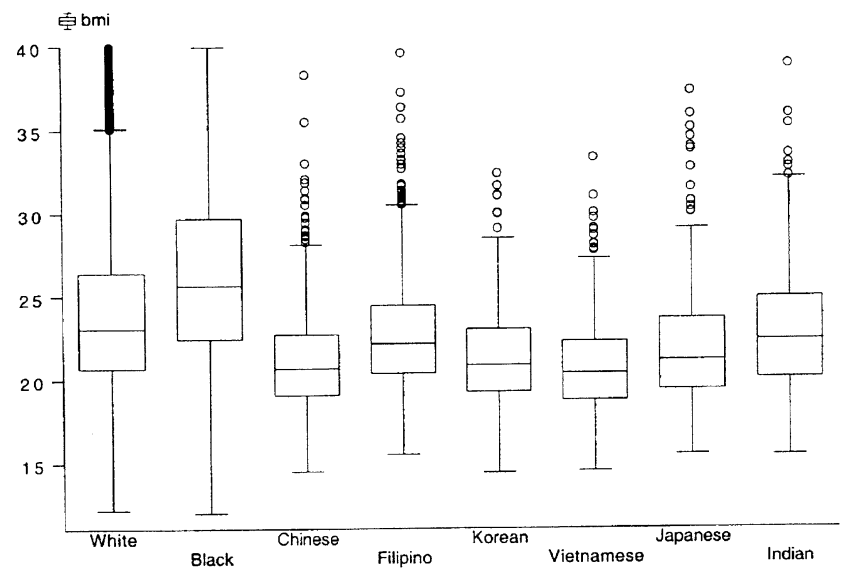

Figure 1 Body mass index for women by race categories from the National Health Interview Survey, 1992-1995. Each box corresponds to the interquartile range of the data, the 25th to 75th percentiles, and the line in the middle is the median. The line above the box extends to the largest data point less than or equal to the 75th percentile plus 1.5 times the interquartile range. The lower line is formed analogously. Observations beyond this are individually plotted. However, the figure does not display values greater than a BMl of 40 .

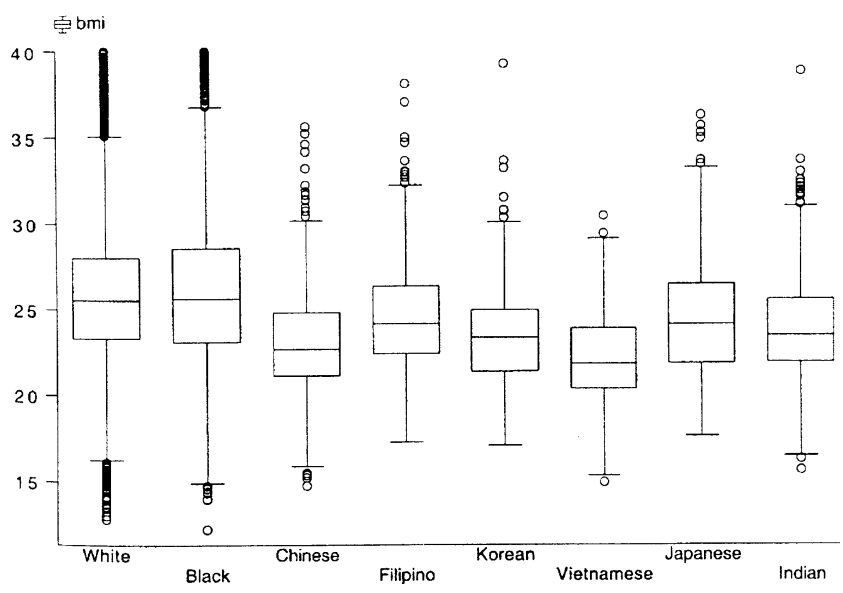

Figure 2 Body mass index for men by race categories from the National Health Interview Survey, 1992-1995. Each box corresponds to the interquartile range of the data, the 25th to 75th percentiles, and the line in the middle is the median. The line above the box extends to the largest data point less than or equal to the 75 th percentile plus 1.5 times the interquartile range. The lower line is formed analogously. Observations beyond this are individually plotted. However, the figure does not display values greater than a BMI of 40 . 